\section{Simultaneous presence of massive chyloperitoneum and chylothorax and its successful management}

\author{
Mukul Bhattarai, Tamer Hudali \\ Department of Internal Medicine, \\ Southern Illinois University, School of \\ Medicine, Springfield, IL, USA
}

\begin{abstract}
The incidence of massive chylous ascites and chylothorax after aortofemoral bypass grafting (AFBG) procedure is unknown. Leakage of chyle results in severe malnutrition and infection which can be life threatening. Because of the rarity of disease, the definitive guideline on management of such extensive accumulation of chyle is lacking. We present a case of a 68year-old gentleman who developed large chylous ascites and chylous pleural effusions causing respiratory distress. His history was significant for aortofemoral bypass graft procedure 8 weeks prior to index presentation. He was successfully managed with chylous fluid drainage, judicious use of diuretics, adherence to a low-fat diet, and supplementation with medium-chain triglycerides.
\end{abstract}

\section{Introduction}

Aortobifemoral bypass grafting (AFBG) has been commonly done procedure in patients with symptomatic or severe aortoiliac occlusive disease (AIOD). ${ }^{1}$ The incidence of incisional hernia and prolonged postoperative ileus secondary to large incision and extensive bowel manipulation in post AFBG procedure has been discussed but, in the current advanced surgical practice, the incidence of significant chylous accumulation both in peritoneal and pleural space is not known. ${ }^{1}$ We present a rare case of very large amount of chylous accumulation in abdomen and lung causing respiratory distress in a patient after 8 weeks of AFBG procedure.

\section{Case Report}

A 68-year-old man with past medical history of hypertension, alcohol abuse, peripheral vascular disease, status post aortobifemoral bypass graft (AFBG) procedure presented to emergency department with abdominal distension and worsening of shortness of breath for past several days. He also reported progressive generalized weakness and lower leg swelling. Upon arrival, his vitals were unremarkable except for heart rate of $110 / \mathrm{min}$. He appeared cachectic (BMI of $18 \mathrm{~kg} / \mathrm{m}^{2}$ ) and was in mild distress. He had significant abdominal distention and bilateral lower extremity pitting edema up to both thighs. His routine lab results were unremarkable except for low albumin of $2 \mathrm{mg} / \mathrm{dL}$. The chest $\mathrm{x}$ ray showed large right pleural effusion. The ultrasound also confirmed a large amount of ascites. Duplex ultrasonography of abdomen revealed no hepatic mass and a patent portal vein with normal directional flow. A transthoracic echocardiogram demonstrated an ejection fraction of $70 \%$ without gross abnormalities. CT scan of chest, abdomen and pelvis consistently showed very large right pleural effusion (Figure 1) and ascites (Figure 2) without evidence of malignancy. He underwent a paracentesis with removal of $5.2 \mathrm{~L}$ of milky fluid (Figure 3). A diagnostic and therapeutic thoracentesis were performed draining $900 \mathrm{~mL}$ of similar milky fluid from the right pleural space.

Fluid analysis revealed significantly elevated triglycerides of $2034 \mathrm{mg} / \mathrm{dL}$ confirming chyloperitoneum or chylous ascites. The ascitic fluid cytology was negative for malignant cells, and the serum quantiferon TB was indeterminate. Pleural fluid analysis also showed highly elevated triglycerides just like that of ascitic fluid. Serum lipid panel constituted total cholesterol of $86 \mathrm{mg} / \mathrm{dL}$, serum triglycerides of $84 \mathrm{mg} / \mathrm{dL}$, and LDL of $43 \mathrm{mg} / \mathrm{dL}$. Based on history of AFBG and excluding the other causes of chyle accumulation, abdominal lymphatic injury was suspected. The surgical history of AFBG was revisited. He underwent AFBG procedure for symptomatic aortoiliac occlusive disease without apparent surgical complication about 8 weeks prior to the index presentation. One month after the procedure, he was treated for right lower extremity incisional wound dehiscence and infection. Exploration of the femoral graft with lavage was done followed by wound vac application.

Review of imaging studies with a radiologist did not reveal any obvious lymphatic duct abnormalities or obstruction. Vascular surgeons were consulted, and the chylous fluid was determined to be from lymphatic trauma while performing AFBG procedure. However, the patient's fluid overload was also attributed to hypoalbuminemia from malnutrition and alcoholic liver disease.
Correspondence: Mukul Bhattarai, Division of General Internal Medicine, Southern Illinois University, School of Medicine, 801 N Rutledge St, Springfield, IL 62702, USA. E-mail: drmukulbhattarai@gmail.com

Key words: chylous ascites, chylothorax, lymphatic injury

Acknowledgments: we would like to thank Adam Roloff, MLIS, from SIU School of Medicine Medical Library for language editing.

Contributions: the authors contributed equally

Conflict of interests: the authors declare no potential conflict of interests.

Conference presentation: part of this paper was selected for poster presentation at the Society of Hospital Medicine conference, Sandiego, CA, USA.

Received for publication: 24 April 2018.

Revision received: 2 June 2018.

Accepted for publication: 2 June 2018.

This work is licensed under a Creative Commons Attribution 4.0 License (by-nc 4.0).

CC Copyright M. Bhattarai and T. Hudali, 2018

Licensee PAGEPress, Italy

Veins and Lymphatics 2018; 7:7520

doi:10.4081/vl.2018.7520

He was started on low dose of furosemide and spironolactone to facilitate the excretion of fluid to reduce the leg swelling and effusions. Dietician helped to manage low fat, high-protein diet containing medium chain fatty acid. His symptoms resolved with fluid drainage (thoracentesis, paracentesis), diuretics, adherence to a lowfat diet, and supplementation with mediumchain triglycerides. He was then subsequently discharged home after 4 days of hospitalization with close vascular surgery follow up. He continued to do well after two months following discontinuation of diuretics as an outpatient, without reaccumulation of chylous fluid.

\section{Discussion and Conclusions}

We presented the uncommon case of large amount of chylous fluid accumulation both in peritoneum and pleura in a 68-yearold man who presented with ascites and pleural effusion. He was found to have very large chylous effusions (both chylous ascites and chylous thorax) 8 weeks following AFBG surgery. 


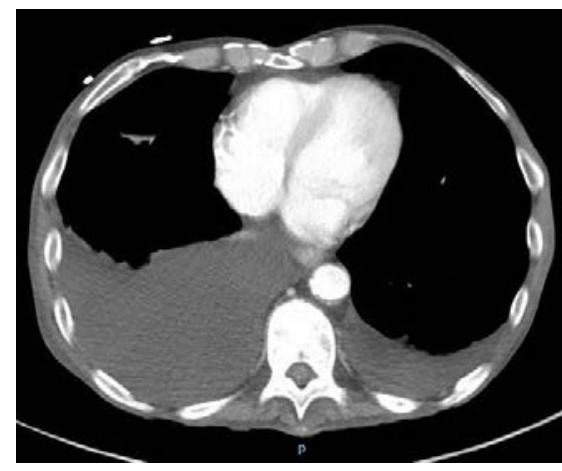

Figure 1. A section of Chest CT showing large right and small left pleural effusion. The right pleural effusion turned out to be large chylothorax.

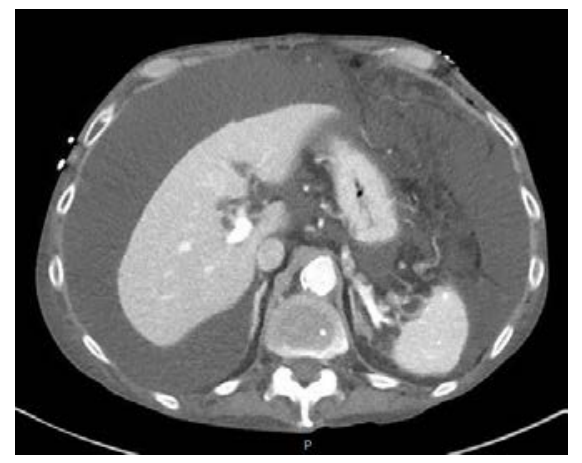

Figure 2. A section of abdominal CT scan showing large ascites. The paracentesis revealed chylous fluid.

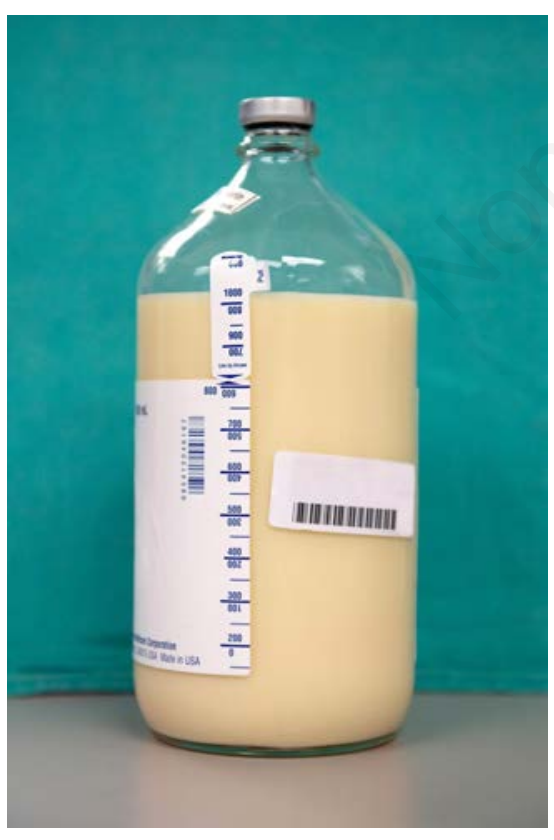

Figure 3. Demonstrating milky (Chylous) fluid draining from paracentesis, similar fluid was also drained from right pleural space.
Usually, the location of chylous effusions in either peritoneal space or pleural space or both depends on site of injury of lymphatic system. ${ }^{2}$ The thoracic duct, a major lymphatic structure, begins at cisterna chyli, which is located intraabdominally anterior to the body of the second lumbar vertebrae to the right side of the aorta. It enters the thorax by crossing the diaphragm at the aortic opening (T12) between the aorta and azygos vein, and crosses the mediastinum at T5 toward the left side. ${ }^{3}$ Disruption of the thoracic duct along its course below T5 can cause a chylothorax. Injury to the intraabdominal portion of the thoracic duct or to the para-aortic or mesenteric lymphatics including cisterna chyli can cause chylous ascites. ${ }^{2}$ Almost $80 \%$ of cases of chylothorax are iatrogenic as a result of oesophagectomy or pneumonectomy. ${ }^{4,5}$ Our patient developed both large volume chylous ascites with chylothorax after aortobifemoral bypass surgery. Intraabdominal lymphatic injury (cisterna chyli or one of its mesenteric tributaries) is likely cause of large chylous effusions in our patient during dissection of the aorta. The translocation of ascitic chyle through a diaphragmatic hiatus may have caused chylothorax. ${ }^{2}$ Chylous effusions are very rare complication of AFBG procedure, specifically in both peritoneal and pleural space. ${ }^{1}$ However, chylous ascites after aortic aneurysm surgery is discussed as a complication but is still rare. . $^{2,5}$

Evidence-based guidelines are lacking to provide the best treatment options in a patient with chylous effusions and ascites because of its rarity. ${ }^{4,5}$ The treatment usually consists of drainage, adherence to a low-fat diet, and supplementation with mediumchain triglycerides, which directly enter the portal system without requiring intestinal lymphatics. ${ }^{2}$ Diuretic may be considered if patient is volume overloaded such as in our case who had bilateral lower extremities edema. ${ }^{6}$ Uncontrolled chyle loss is associated with significant morbidity and mortality from malnutrition, immunosuppression and infection. ${ }^{4,7}$ Chylous effusions have high mortality of up to $50 \%$, but the conservative management has shown promise to reduce the complication significantly., ${ }^{2,7}$ The conservative treatment with total parenteral nutrition (TPN) favors over oral intake of low fat diet with medium chain triglyceride if chylous leaks are extensive and patient is not able to meet the nutrition requirement by oral intake. If the milky effusion persists for several days despite continuous TPN, the somatostatin, or its synthetic analogue octreotide, is a potential alternative to surgical management of chyle leaks. ${ }^{8,9}$ The somatostatin decreases the intestinal absorption of fats via the specific receptors, resulting in decrease in triglyceride concentration in the thoracic duct which causes attenuation of the lymph flow in the major lymphatic channels. ${ }^{9}$ Such therapy is found to be quite effective in resolving neck chylous fistulas. ${ }^{8}$ Only in refractory postoperative chylothorax and chylous ascites, lymphangioscintigraphy is considered for further evaluation and treatment. ${ }^{2,10}$ Therapeutic embolization using acrylate glue and platinum coils may be required. ${ }^{7}$ The transjugular intrahepatic portosystemic shunt is considered in patients with cirrhotic patients associated with chylous ascites. ${ }^{11}$ However, only $0.5 \%-1 \%$ of all cases of cirrhosis $\square$ related ascites are associated with chylous accumulation. ${ }^{11}$ The traditional surgery such as peritoneovenous shunting is rarely done because of high risk of severe infection, electrolyte disturbances, disseminated intravascular coagulopathy, small bowel obstruction and air embolism. ${ }^{11}$

In conclusion, any patient who develops pleural effusion and ascites after an AFBG procedure should have chylous ascites and chylothorax included in the differential diagnosis. This report also adds to the current literature about the approach to management of chylothorax and chylous ascites when definitive guidelines for management is lacking. The conservative approach is the best initial modality to treat even very large chylous fluid accumulation in both pleural and peritoneal space that occurs from AFBG procedure complication.

\section{References}

1. Klem TMAL, van der Ham AC, de Smet AAEA, et al. Hand Assisted Laparoscopic Surgery of Aortoiliac Occlusive Disease: Initial Results. Eur J Vasc Endovasc Surg 2006;32:639-44.

2. Ly TY, Fowler RA. Chylothorax and Chyloperitoneum. N Engl J Med 2006; 354:879.

3. Thoracic Duct Anatomy: Overview, Gross Anatomy, Microscopic Anatomy. 2017 Dec 7; Available from: https:// emedicine.medscape.com/article/19701 45-overview? pa=a6c8zmj N6NVHBy3 goM471OyOVimOdqWaLV3SAPF\%2F 0zD8wWr3xh1HNOXLZdzkGXgKVrJ xKJt4DRD8mxYr6kYfOw\%3D\%3D

4. Nair SK, Petko M, Hayward MP. Aetiology and management of chylothorax in adults. Eur J Cardio-Thorac Surg Off J Eur Assoc Cardio-Thorac Surg 2007;32:362-9.

5. Olthof E, Blankensteijn JD, Akkersdijk GJM. Chyloperitoneum following abdominal aortic surgery. Vascular 
2008;16:258-62.

6. Cirrhotic Ascites [Internet]. [cited 2018 May 25]. Available from: http://www.clevelandclinicmeded.com/ medicalpubs/diseasemanagement/hepatology/complications-of-cirrhosisascites/

7. Allison S, Rainey M, Aarabi S, Padia SA. Traumatic laceration of the cisterna chyli treated by lymphangiography and percutaneous embolization. Cardiovasc Intervent Radiol 2014;37:267-70.
8. Swanson MS, Hudson RL, Bhandari N, et al. Use of Octreotide for the Management of Chyle Fistula Following Neck Dissection. JAMA Otolaryngol Neck Surg 2015;141:7237.

9. Karaca S, Gemayel G, Kalangos A. Somatostatin treatment of a persistent chyloperitoneum following abdominal aortic surgery. J Vasc Surg 2012; 56:1409-12.

10. Kawasaki R, Sugimoto K, Fujii M, et al.
Therapeutic effectiveness of diagnostic lymphangiography for refractory postoperative chylothorax and chylous ascites: correlation with radiologic findings and preceding medical treatment. AJR Am J Roentgenol 2013;201:65966.

11. Lizaola B, Bonder A, Trivedi HD, et al. Review article: the diagnostic approach and current management of chylous ascites. Aliment Pharmacol Ther 2017;46:816-24. 